\title{
4. Imagen cardiovascular
}

\subsection{Identificación de trompa de Eustaquio redundante en paciente con flutter auricular mediante ecocardiografía}

Esparza Rafael, Cortés Aguirre Mauricio, Flores Silva Francisco Javier Hospital Regional Monterrey ISSSTE.

Tipo de estudio: Reporte de casos clínicos con revisión de fuentes

Introducción: La trompa de Eustaquio o red de Chiari es un remanente de la válvula del seno venoso derecho, presente en $2 \%$ de la población, puede no dar síntomas o puede asociarse con fibrilación auricular, endocarditis y formación de embolismos. Descripción del caso: Mujer de 61 años APNP tabaquismo positivo, APP DM2 (+) HTA (+). Inicia hace 10 meses, con palpitaciones, disnea y lipotimia, se detecta flutter auricular, se dio manejo antiarrítmico, persiste con sintomatología y se envía al Servicio de Electrofisiología para mapeo y ablación. Se coloca ecocardiograma intracardiaco. Metodología: Catéter en seno coronario y en aurícula derecha, catéter de ablación del istmo cavotricuspídeo, se observa trompa de Eustaquio redundante, se realiza bloqueo bidireccional del istmo cavotricuspídeo, y termina procedimiento sin complicaciones. Se programa ecocardiograma transesofágico encontrando: estructura ondulante, extensa y altamente móvil correspondiente a válvula de Eustaquio, en la desembocadura de la vena cava inferior, se inyecta solución salina. Resultados: Sin observarse embolismo paradójico. Discusión: Dentro del diagnóstico diferencial deben descartarse tumores, vegetaciones y trombos, existen distintos métodos diagnósticos como es el ecocardiograma transesofágico y en este caso el ecocardiograma intracardiaco. Conclusión: La red de Chiari es un remanente del sistema venoso que debido a que generalmente no da síntomas, es subdiagnosticado, por lo que debería buscarse durante los estudios de gabinete, debido a la posible asociación con otras patologías o complicaciones.

\subsection{Cardiomiopatía dilatada congénita secundaria a falta de compactación del ventrículo izquierdo}

González Zazueta Rafael, González Olvera Jimena Alejandra, Jiménez Santos Moisés, González Montecinos Alejandro Becerril, Lupercio Mora Karina, Almeyda Gutiérrez Eduardo
Hospital de Cardiología del Centro Médico Nacional Siglo XXI.

Tipo de estudio: Reporte de casos clínicos con revisión de fuentes

Introducción: La falta de compactación de ventrículo izquierdo es una cardiomiopatía no clasificada caracterizada por una capa endocárdica extremadamente gruesa con trabeculaciones prominentes y una capa epicárdica delgada. A pesar de que puede presentarse de forma aislada, también se puede asociar a ciertas cardiopatías como la anomalía de Ebstein y tetralogía de Fallot, además de ciertos desórdenes neuromusculares. Descripción del caso: Mujer de 20 años que acude por disnea progresiva, cardiomegalia e hipertensión venocapilar en radiografía. Ecocardiograma: dilatación biventricular, fracción expulsión 21\%, TAPSE $10 \mathrm{~mm}$, insuficiencia mitral y tricuspídea severas, trabeculaciones con flujo en cuatro caras en eje corto, strain-7.9\%. Resonancia magnética: incremento de trabeculación en ambos ventrículos, relación $\mathrm{MNC}$ / MC > 2.4 y miocardio no compacto en ventrículo izquierdo de $50 \%$. Metodología: A través de método booleano se realizó búsqueda de términos MeSH noncompaction cardiomyopathy [and] diagnosis en las bases de datos con repositorios de evidencia científica arbitrada indizada (PubMed) y no indizadas (Google Scholar). Resultados: LA ESC y AHA actualmente no cuenta con criterios diagnósticos para la detección de la miocardiopatía no compactada, usándose en la actualidad distintos criterios ecocardiográficos de Chin, Jenni y Stollberger, con alta sensibilidad y baja especificidad, complementándose abordaje con criterios de resonancia magnética de Petersen y Jaquier, aumentando la sensibilidad y especificidad > 93\%. Discusión: A pesar de no contar con estándar de oro para detección de miocardiopatía no compactada, en este caso se cuenta con criterios positivos por ecocardiograma y resonancia magnética, concluyéndose el diagnóstico; en esta patología la presentación clínica suele ser arritmias, embolismos y falla cardiaca, por lo que se debe tratar de acuerdo con las guías de falla cardiaca y valorar anticoagulación.

\subsection{Elección terapéutica en el manejo de la $\mathrm{CI}$ en la mujer; trascendencia estudio de gated-SPECT}

Olivares García Pedro Israel, Puente Barragán María del Carmen, Martínez Escobar María del Carmen

Centro Médico Nacional 20 de Noviembre, ISSSTE.

Tipo de estudio: Estudio pronóstico
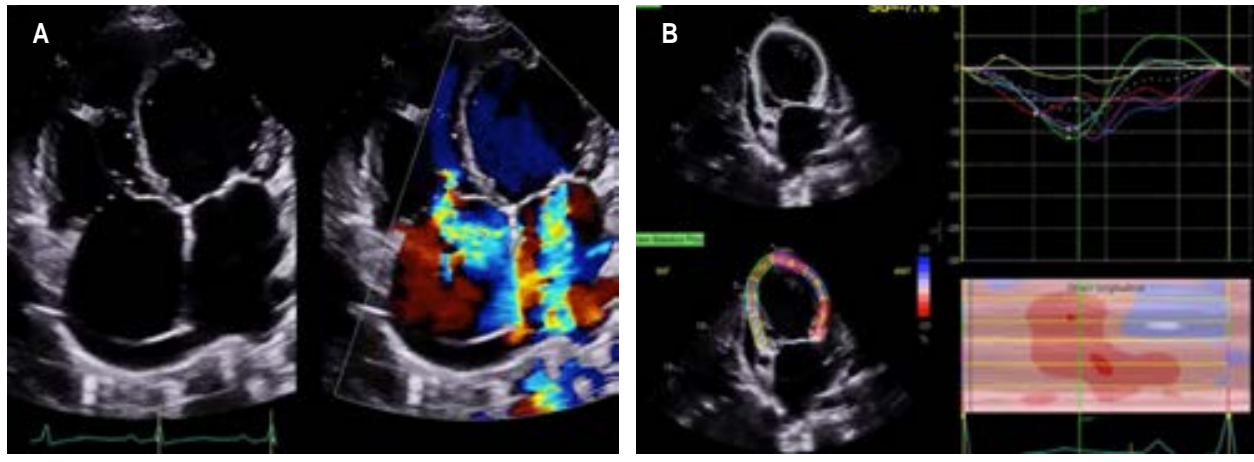

Figura 4.2.1:

Imágenes por ecocardiograma transtorácico. A) Eje cuatro cámaras con evidencia de dilatación biventricular y auricular, con importantes recesos endomiocárdicos, se observan además insuficiencias valvulares severas al aplicar Doppler color. B) Eje dos cámaras con strain global longitudinal de - 7.1\%, obsérvese la mayor afección hacia el ápex y pared anterior. 

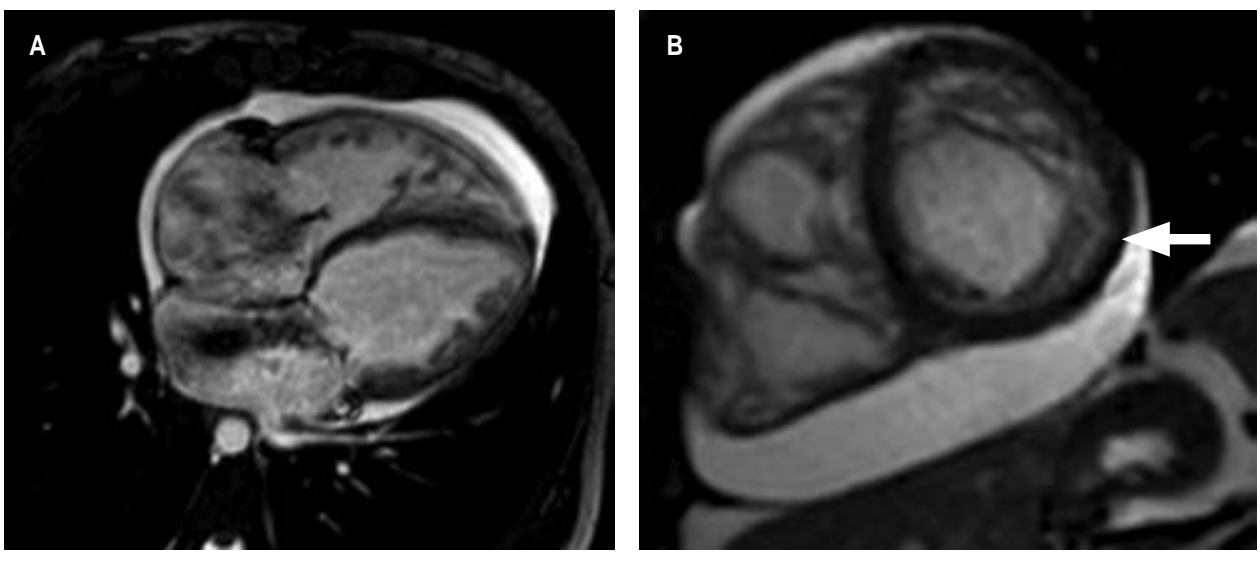

Figura 4.2.2:

Imágenes por resonancia magnética. A) Eje cuatro cámaras con trabeculaciones evidentes en ambos ventrículos, se puede observar además insuficiencia tricuspídea y mitral severa. B) Eje corto donde se observa la relación $\mathrm{MN} / \mathrm{MC}$ $>2.4$ (flecha) diagnóstica de miocardiopatía no compactada.

Introducción: La cardiopatía isquémica $(\mathrm{Cl})$ es una de las principales causas de muerte en mujeres. Se asocia con otros factores de riesgo cardiovascular (FRCV), y subutilización de métodos diagnósticos. Un método diagnóstico de imagen no invasiva es el estudio de perfusión miocárdica Gated-SPECT y es útil en la evaluación y toma de decisiones terapéuticas en mujeres con $\mathrm{Cl}$. Objetivo: Determinar el valor agregado del gated-SPECT (g-SPECT) en la decisión terapéutica en mujeres con $\mathrm{Cl}$. Metodología: Estudio restrospectivo, evaluamos result g-SPECT 120 mujeres, diagnóstico de Cl. Criterios exclusión: cardio estructural, miocardiopatía hipertrófica/dilatada, inestabilidad hemodinámica. Características clínicas: edad, ECV previa, y FRCV. Gated-SPECT: protocolo de un día sestamibi-Tc99m. Imágenes adquiridas en una gamma-cámara cardiodedicada y evaluadas subjetivamente en 17 segmentos. Riesgo postprueba: bajo, inter y alto. Análisis estadístico: medias \pm DE y porcentajes; correlación de Pearson. Resultados: $64 \pm 10$ años. FRCV: 90\% postmenopáusicas; 68\% HA, 43.3\% DM2, 50.8\% dislip, 28.6\% tab y $4.1 \%$ ERC. Indi g-SPECT: infarto antiguo $34.4 \%$, IAM 12.5\%, AC $51.6 \%$, y AI 9.8\%. Resul. g-SPECT: 15\% normal, 44\% riesbajo, 33\% inter y 18\% alto. Cateterismo: 49 (40\%); 9 (19\%) g-SPECT normal, 10 (20\%) riesbajo, 25 (51\%) inter y 5 (10\%) alto. TX: PCI 43 (87.8\%); 9 (20.9\%) sin riesgo, 9 (20.9\%) bajo, $22(51.2 \%)$ inter, y 3 (7\%) alto. GABA: 6 (12.2\%); 1 (16.7\%) riesbajo, 3 (50\%) inter, 2 (33.3\%) alto. Existió correlación significativa entre el riesgo postprueba (inter-alto) y necesidad de cateterismo $r=0.758, p=0.035$. Discusión y conclusiones: El gated-SPECT confiere valor agregado significativo en la toma de decisiones terapéuticas en mujeres con $\mathrm{Cl}$, y reduce el número de procedimientos diagnósticos invasivos innecesarios.

\subsection{Síndrome de Takotsubo durante inducción anestésica en paciente masculino: reporte de caso}

Borges López Jesús Samuel, Rizo Cortedano Darwin Saúl,

Camacho Bernal Raúl, González Maciel Javier

Hospital General de México «Dr. Eduardo Liceaga».

Tipo de estudio: Reporte de casos clínicos con revisión de fuentes

Introducción: El síndrome de Takotsubo, también llamado «corazón roto» o cardiomiopatía por estrés, se presenta en menos del 1\% de pacientes con sospecha de síndrome coronario agudo y predomina en mujeres postmenopáusicas y es originado por una descarga simpática excesiva multifactorial que provoca espasmo coronario y disfunción ventricular. Descripción del caso: Hombre de 44 años

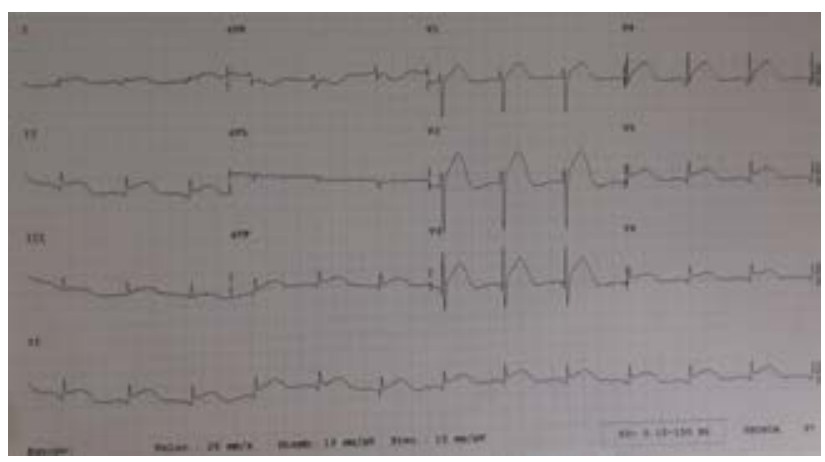

Figura 4.4.1.

programado para aseo quirúrgico de úlcera sacra infectada, durante inducción anestésica tuvo dolor anginoso típico, evidenciando en electrocardiograma elevación del ST en cara inferolateral, con elevación de TI en 7,189 indicando fibrinolisis presentando ritmo nodal que ameritó colocación de marcapasos transvenoso, realizando angiografía sin evidencia de lesiones, observando en ventriculografía datos de síndrome de Takotsubo. Resultados: El síndrome de Takotsubo es una falla cardiaca aguda transitoria descrita por primera vez en la población japonesa en 1990, siendo cada vez más frecuentes los reportes asociados al periodo perioperatorio. Su diagnóstico se basa en características clínicas, electrocardiográficas y bioquímicas, con evidencia de discinesia apical e hipercinesia basal en la ventriculografía con ausencia de lesiones coronarias en la angiografía. Discusión: En el entorno perioperatorio existen múltiples desencadenantes de estrés, por lo que es posible encontrarnos ante este síndrome. El caso anterior cobra importancia al ser la inducción anestésica el desencadenante de dicho cuadro, presentándose además en un hombre, lo cual es poco habitual, debiéndose brindar un tratamiento oportuno y vigilar las posibles complicaciones como arritmias y choque cardiogénico.

\subsection{Fístula coronaria a arteria pulmonar vía anillo arterial de Vieussens $y$ aneurisma gigante}

González Gurrola Erwing Enrique

Hospital de Cardiología del Centro Médico Nacional Siglo XXI, IMSS.

Tipo de estudio: Reporte de casos clínicos con revisión de fuentes 
Figura 4.4.2.
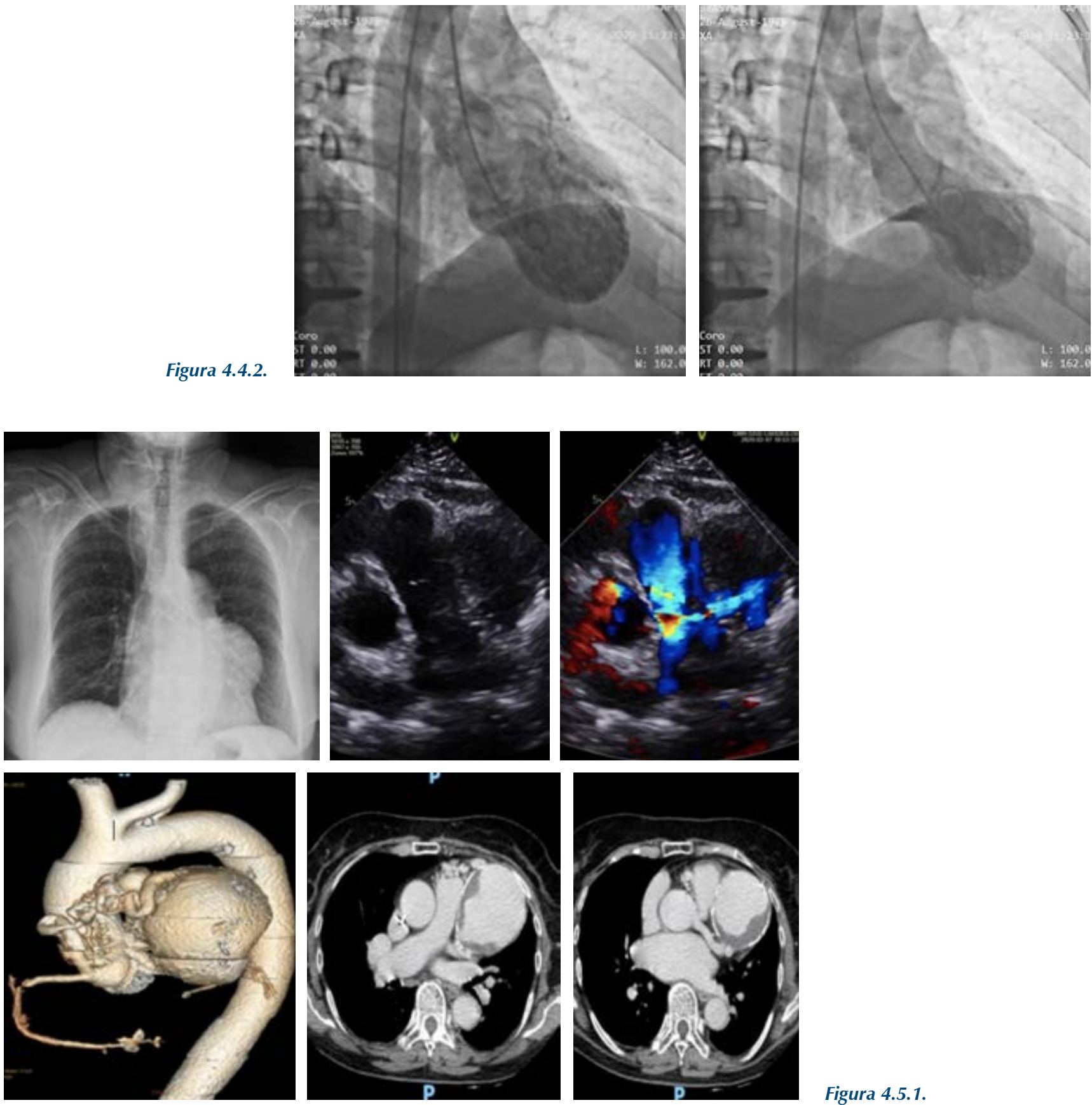

Figura 4.5.1.

Introducción: La fístula congénita de arteria coronaria a arteria pulmonares (FACP) es una anomalía congénita poco común, caracterizada por conexión anormal que une directamente una o más arterias coronarias a tronco arteria pulmonar. La conexión vía anillo arterial de Vieussens es muy raro, con pocos reportes en la literatura con presentación como masa mediastínica. Descripción del caso: Mujer de 67 años, referida por imagen radioopaca paracardiaca izquierda y dolor torácico atípico; exploración soplo paraesternal izquierdo continuo II/VI. Abordaje con multiimagen: evidencia FACP con dilatación aneurismática $(84 \mathrm{~mm})$ y trombo, comunica arteria pulmonar vía anillo Vieussens. Tratamiento percutáneo con embolización (coils) con un resultado insatisfactorio. Tratamiento quirúrgico con ligadura y aneurisectomía no exitoso. Metodología: Se realizó búsqueda en MEDLINE y Scopus con los términos coronary artery to pulmonary artery fistula, coronary fistula aneurysm, coronary fistula via arterial Vieussens ring, surgical treatment, percutaneous treatment. Se buscaron artículos relevantes en inglés publicados entre el 1 de enero de 1992 y el 30 de julio de 
2020. Se identificaron publicaciones adicionales mediante revisión bibliográfica. Resultados: Se encontraron 25 artículos a los que se hace referencia en esta revisión. Diámetro de aneurisma reportado en serie de casos entre 20 a 70 mm; sólo dos reportes de caso vía anillo arterial Vieussens; presentamos caso con aneurisma gigante $84 \mathrm{~mm}$ como manifestación de masa mediastínica, el más grande reportado hasta ahora. Discusión: En conclusión, la FACP es el tipo más común de fístula de arteria coronaria que se encuentra incidentalmente en estudios de imagen. Debe considerarse como una causa potencial en el diagnóstico diferencial de abordaje de masas mediastínicas. Se reporta tamaño aneurisma coronario ( $>80$ $\mathrm{mm}$ ) y morfología de trayectos de FACP como posibles predictores de mal pronóstico quirúrgico.

\subsection{Impacto de la viabilidad miocárdica determinada por PET CT con FDG-18 de pacientes sometidos a revascularización}

Pérez Siller Grecia Rosangela, Martínez-

Escobar María Carmen, Ávalos-Ríos Miguel

ISSSTE.

Tipo de estudio: Estudio pronóstico

Introducción: Existen estudios demostrando el efecto positivo de la revascularización guiada mediante la determinación de viabilidad miocárdica en angina crónica estable. El estudio de PET-CT marcado con FDG-18 permite seleccionar a los pacientes que serán candidatos a revascularización miocárdica (percutánea o quirúrgica) la interrogante es si la presencia de viabilidad miocárdica se relaciona con mayor o menor eventos cardiovasvulares mayores (MACE). Objetivo: Identificar si los pacientes sometidos a revascularización miocárdica con tejido viable presentan más MACE que los pacientes revascularizados sin tejido viable. Metodología: Es un estudio observacional, descriptivo, transversal. Pacientes con agina crónica estable sintomáticos y asintomáticos con estudio de viabilidad miocárdica con PET CT marcado con FDG-18 desde inicios del 2016 hasta diciembre del 2019 realizado en Servicio de Medicina Nuclear Cardiaca, se realiza seguimiento (mínimo de un año) identificando MACE. Resultados: Se incluyeron 41 pacientes. Los eventos de MACE en población revascularizada sin tejido viable fueron tres pacientes con infarto agudo al miocardio, dos pacientes con necesidad de revascularización y dos pacientes con desenlace mortal por etiología cardiovascular. Los eventos de MACE en población revascularizada con tejido viable fueron un paciente con desenlace mortal por etiología cardiovascular. Discusión y conclusiones: Los resultados obtenidos sugieren que siempre que sea factible es mandatorio realizar un estudio de viabilidad miocárdica debido a las implicaciones terapéuticas y pronósticas en pacientes con enfermedad coronaria.

\subsection{Miocarditis aguda: reporte de caso}

Pérez Roa Herlich Francisco,* Soto González Juan Ignacio, ${ }^{\ddagger}$ Hernández Bravo Mariana Jazmín, ${ }^{\ddagger}$ García Jiménez Yoloxóchitl, ${ }^{\ddagger}$ Sánchez Martínez María Isabel, ${ }^{\ddagger}$ Durán Sáinz Victor Manuel, ${ }^{\ddagger}$ Marín Rendón Sadoc ${ }^{\ddagger}$

* Instituto Mexicano del Seguro Social. ` Unidad Médica de Alta Especialidad No. 14 del CMN «Adolfo Ruiz Cortines», Veracruz, Ver. Tipo de estudio: Reporte de casos clínicos con revisión de fuentes

Introducción: La miocarditis afecta a individuos de todas las edades, con presentaciones clínicas heterogéneas que oscilan entre angina o palpitaciones con cambios electrocardiográficos transitorios. La presentación más común es infarto de miocardio con arterias
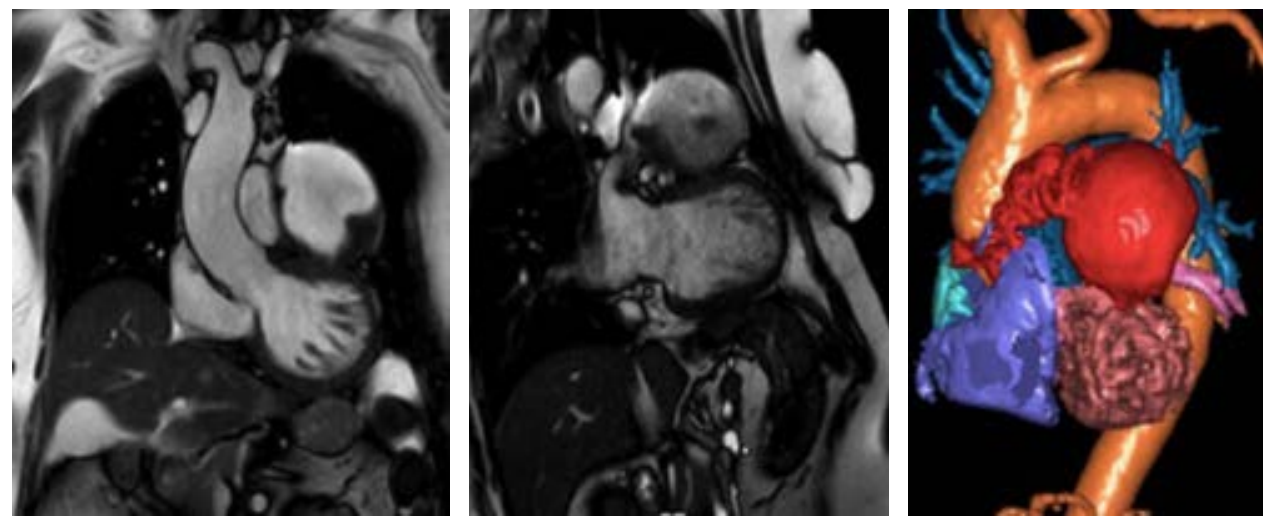

Figura 4.5.2.
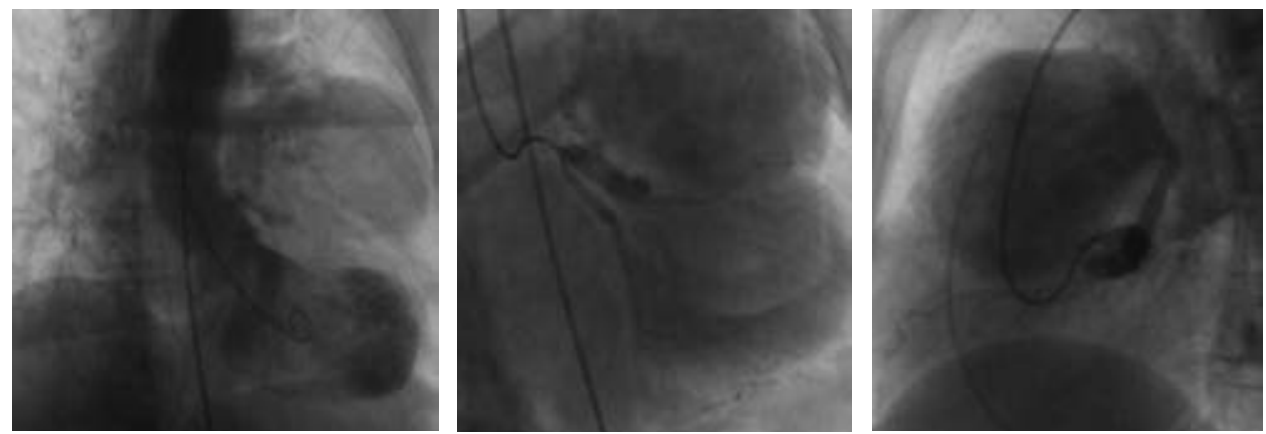
Figura 4.7.1:

Cateterismo cardiaco diagnóstico. A) Proyección angiográfica oblicua anterior izquierda caudal, donde se observa la arteria descendente anterior y circunfleja sin lesiones. B) Proyección angiográfica oblicua anterior izquierda craneal, donde se observa fístula pequeña de la coronaria derecha a la aurícula derecha.
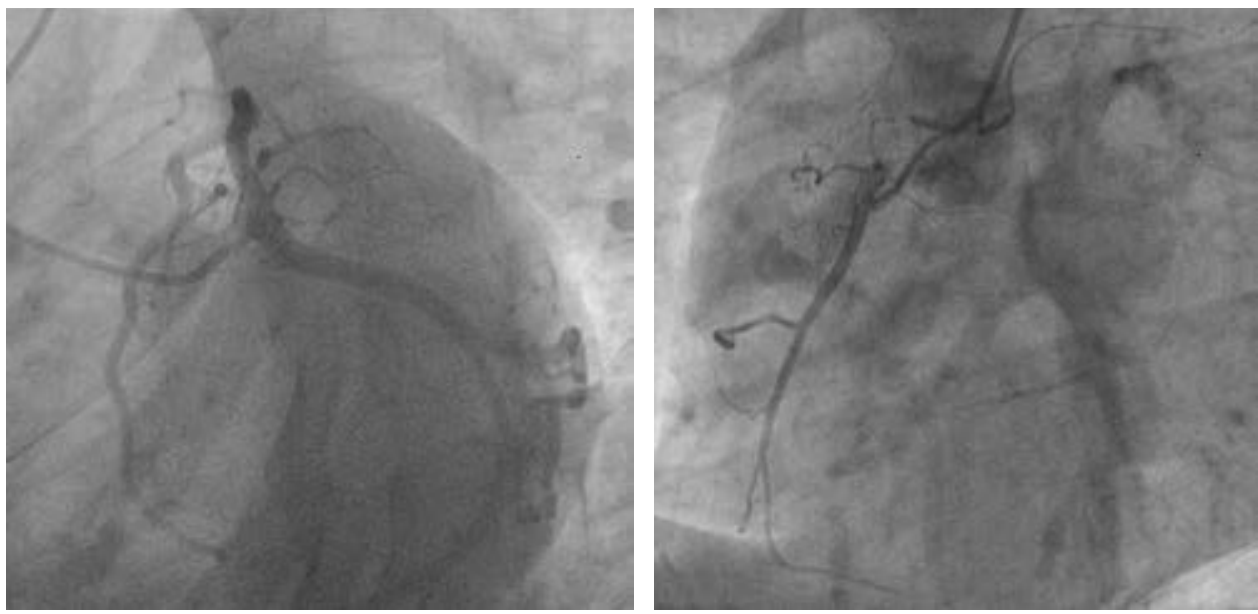

coronarias normales. El ecocardiograma es la primera línea de diagnóstico sin hallazgos específicos, requiriendo resonancia magnética cardiaca o tomografía con emisión de positrones para el diagnóstico. Descripción del caso: Hombre de 43 años, cuadro clínico de dolor torácico opresivo, diaforesis, con electrocardiograma evidenciando desnivel positivo del ST lateral bajo y ecocardiograma con alteraciones de movilidad segmentaria, procediendo a cateterismo cardiaco sin presentar lesiones en coronarias (Figura 4.7.1), estabilizando y complementando con resonancia magnética cardiaca sugerente de miocarditis (Figura 4.7.2). Metodología: Se realiza una búsqueda sistemática y exhaustiva de todos los artículos indexados del 2018 al 2020 disponibles en la base de datos PubMed-Medline con la estrategia de búsqueda implementando la palabra Myocarditis, dando relevancia al factor de impacto. Resultados: La miocarditis es la enfermedad inflamatoria del miocardio, diagnóstico establecido por criterios histológicos, inmunológicos e inmunohistoquímicos; sin embargo, con pobre relación diagnóstica de la biopsia endomiocárdica (9 a 16\%), por lo que la complementación diagnóstica por criterios de Lake-Louis ha alcanzado una sensibilidad de $81 \%$ y especificidad de $71 \%$; por esto la justificación de dicho estudio en el protocolo diagnóstico. Discusión: Considerando la heterogeneidad de la presentación clínica y la limitación de los estudios no invasivos de imagen para el diagnóstico de la miocarditis, el ecocardiograma, pese a ser la primera línea, solo apoya como guía y se sugiere la resonancia magnética cardiaca en búsqueda intencionada de los criterios de Lake-Louis, logrando el diagnóstico oportuno y seguimiento clínico cercano.

\subsection{Evidencia de isquemia mediante gated- SPECT en disfunción protésica}

Ávalos Ríos Javier Miguel, Martínez Escobar María del Carmen, Pérez Siller Grecia Rosangela, Puente Barragán Adriana, González Gutiérrez Carlos, Mendoza Avellán Letty María Centro Médico Nacional 20 de Noviembre, ISSSTE.

Tipo de estudio: Reporte de casos clínicos con revisión de fuentes

Introducción: El dolor torácico es un reto diagnóstico para el clínico, la imagen con radionúclidos puede ser útil en la identificación de aterosclerosis coronaria subclínica para la prevención primaria, IAM o muerte súbita. El gated-SPECT es el método clínico

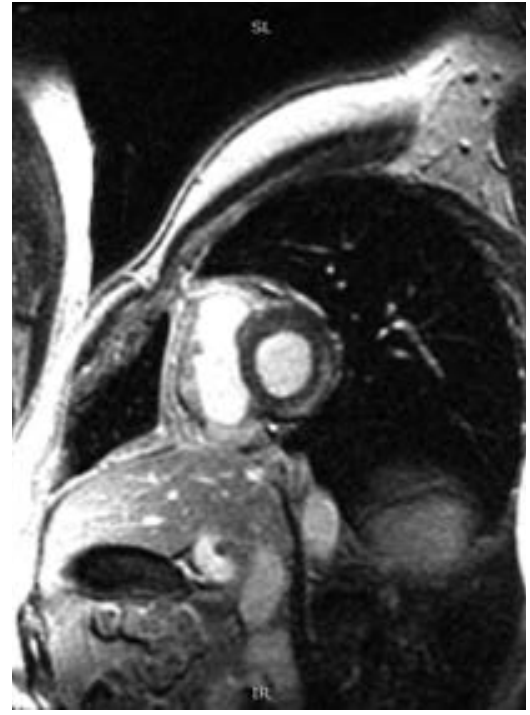

Figura 4.7.2:

Resonancia magnética cardiaca con edema y realce tardío en la pared lateral, tercio medio y apical del ventrículo izquierdo sugerente de miocarditis.

principal para la evaluación de la isquemia miocárdica, y también permiten obtener imágenes sólidas de pacientes con patología múltiple. Descripción del caso: Hombre de 60 años. IAM hace nueve años, dos hemoductos y sustitución aórtica. Presenta angina y disnea. Electrocardiograma (infradesnivel ST V3-V6, DII, DIII y AVF). Gated-SPECT, infarto no transmural, isquemia moderada en ápex, pared anterior e isquemia severa inferolateral. Cateterismo, hemopuentes permeables sin indicación de intervencionismo. Ecocardiograma, aumento de gradientes en válvula protésica (Gmax $65 \mathrm{mmHg}$, medio $32 \mathrm{mmHg}$ ). Metodología: Se realizó búsqueda acerca de la valoración de isquemia en pacientes con sustitución valvular aórtica con estudio de perfusión aórtica gated-SPECT en distintos motores de búsqueda (PubMed, Google Scholar). Resultados: En este caso observamos valoración de un paciente con angina, quien cursa con disfunción protésica y hemoductos permeables; la disfunción valvular y consecuencias hemodinámicas ocasiona este desbalance entre la demanda y suministro de oxígeno e inducir isquemia miocárdica aun en reposo en el 


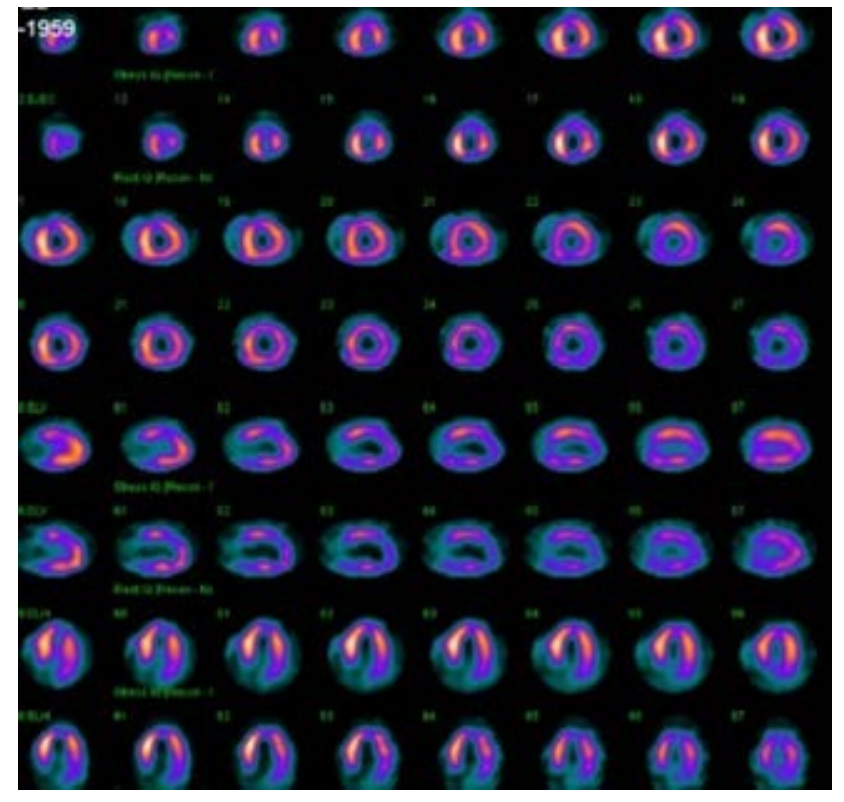

Figura 4.8.1: Estudio de perfusión miocárdica con defecto de perfusión en reposo.

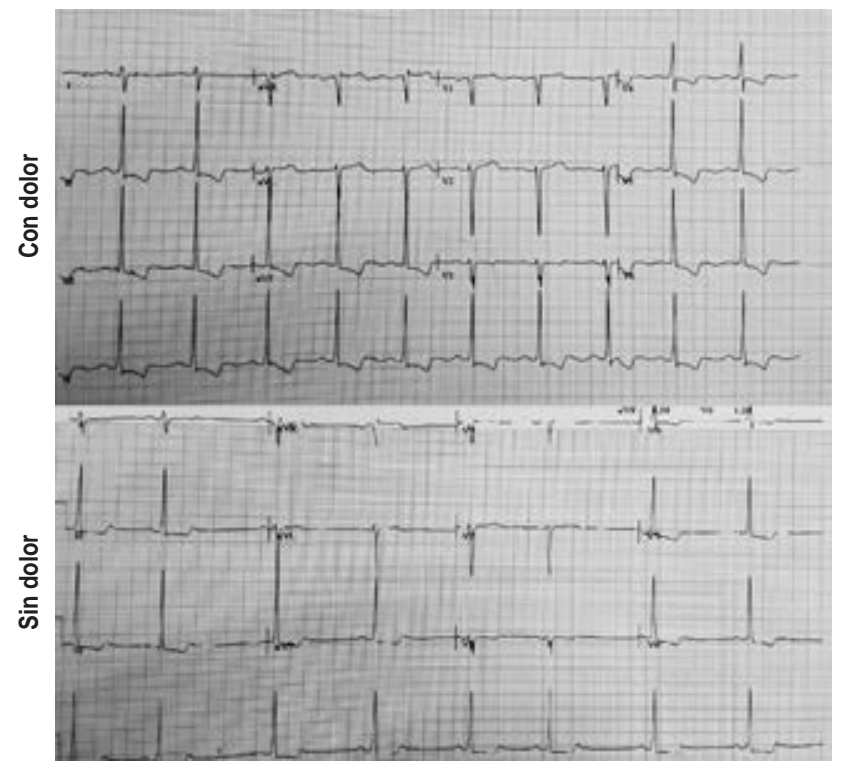

Figura 4.8.2.

caso presentado, la isquemia puede ocasionar síncope y muerte súbita. Discusión: Dentro de los métodos diagnósticos recomendados para pacientes con dolor torácico se encuentra el SPECT, el cual es seguro, no invasivo, preciso y costo-efectivo para valorar enfermedad arterial coronaria. La decisión terapéutica debe ser orientada con métodos de imagen no invasiva, para evaluar y dirigir el tratamiento a métodos mínimamente invasivos.

\subsection{Rol del gated-SPECT en la evaluación de la cardiomiopatía por sobrecarga de hierro}

Ávalos Ríos Javier Miguel,* Martínez Escobar María Del Carmen,*

Puente Barragán Adriana,* Olivares Pedro Israel,*

Pérez Siller Grecia Rosangela, ${ }^{\ddagger}$ Mendoza Avellán Letty María,*

González Gutiérrez Carlos*

* Centro Médico Nacional 20 de Noviembre, ISSSTE. ${ }^{*}$

Hospital Regional «Dr. Valentín Gómez Farías».

Tipo de estudio: Reporte de casos clínicos con revisión de fuentes

Introducción: La miocardiopatía por depósito de hierro es una causa de insuficiencia cardiaca en pacientes con hemocromatosis, originando miocardiopatía restrictiva o dilatada. Se define como la presencia de disfunción sistólica o diastólica secundario a deposición de hierro en corazón, generando fibrosis intersticial, independiente de otros procesos concomitantes. Descripción del caso: Hombre de 40 años, padece cirrosis hepática secundaria a hemocromatosis con hipertensión portal. Ecocardiograma, evidencia disfunción diastólica grado III, incremento de presiones de llenado, insuficiencia mitral severa, Carpentier tipo III B. Eco-dobutamina positiva para isquemia. Perfusión miocárdica gated-SPECT con defecto de perfusión en ápex, pared inferior y región inferolateral fija, ventrículo derecho dilatado. Tc99 PYP negativo para proceso infiltrativo. Metodología: Se realiza búsqueda de información acerca del uso de gated-SPECT y PYP a descartar patología infiltrativa en pacientes con hemocromatosis en motores de búsqueda (PubMed), con palabras relacionadas con hemocromatosis, uso de pirofosfato para descartar cardiomiopatía por hemocromatosis. Resultados: La mortalidad por enfermedad coronaria e insuficiencia cardiaca es alta en los pacientes postrasplante hepático, tratamiento considerado en este caso, por lo que parte del abordaje diagnóstico incluye pruebas de estratificación en pacientes en los que se debe descartar cardiopatía

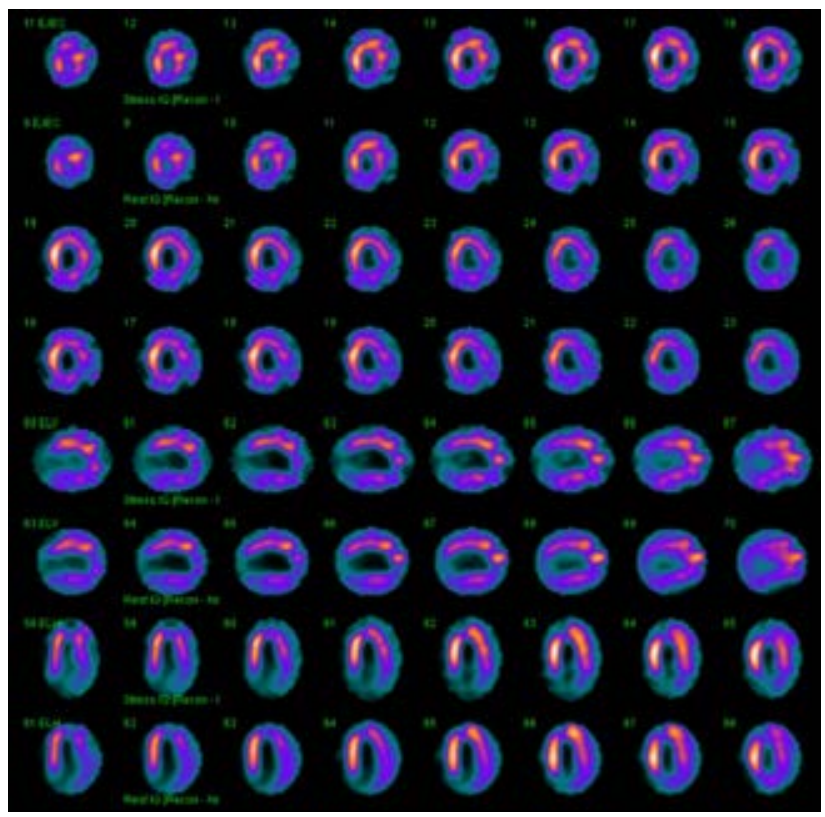

Figura 4.9.1: Estudio de perfusión miocárdica con defecto de perfusión sin reversibilidad de fibrosis, ventrículo izquierdo dilatado. 
isquémica concomitante y el uso de Tc99-PYP indicado para descartar otra etiología infiltrativa. Discusión: El realizar el diagnóstico diferencial para descartar cualquier otra cardiomiopatía infiltrativa es importante al momento de indicar el tratamiento, el estudio de perfusión miocárdica gated-SPECT es importante para definir isquemia como etiología de la insuficiencia cardiaca, establecer pronóstico; evaluar la función cardiaca, indicar sitios sugerentes de fibrosis cardiaca, sincronía ventricular, orientando el tratamiento.

\subsection{Infarto del miocardio inferior por una arteria descendente anterior superdominante. Reporte de caso}

Gallardo Huitrón Judd, García Jiménez Yoloxóchitl, Márquez López Jesús Sinue, Soto González Juan Ignacio, Rascón Sabido Rafael, Marín Rendón Sadoc

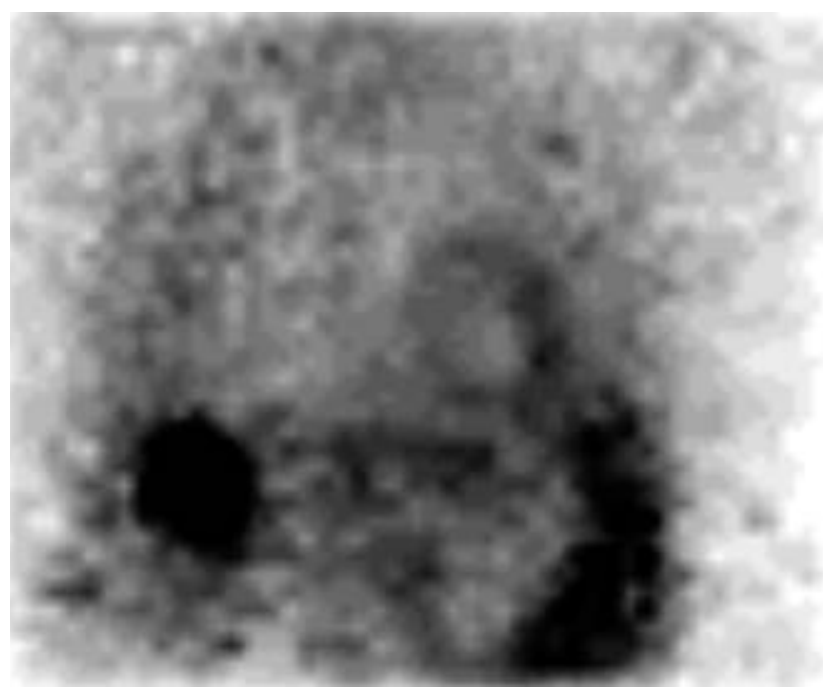

Figura 4.9.2: Estudio de cardiología nuclear rastreo TC99m PYP escala de Perugini grado 1.
Unidad Médica de Alta Especialidad No. 14 del CMN

«Adolfo Ruiz Cortines», Veracruz, Ver.

Tipo de estudio: Reporte de casos clínicos con revisión de fuentes

Introducción: La dominancia coronaria es una variante anatómica, importante por el abordaje terapéutico y pronóstico. Las anomalías congénitas de las arterias coronarias son raras, con una incidencia del 0.3 al 1\%. La irrigación de la cara posterior e inferior por una descendente anterior superdominante es extremadamente rara. Descripción del caso: Mujer de 62 años con angina en reposo, cambios eléctricos de isquemia inferior y lateral baja con Tnl positiva, se concluye infarto sin elevación del ST inferolateral, realizándose cateterismo cardiaco y documentando arterias coronarias sin lesiones angiográficas obstructivas con arteria descendente anterior superdominante. FEVI 55\% hipocinesia inferobasal. Se optimizó tratamiento farmacológico antiisquémico con evolución satisfactoria. Metodología: Se realizó una revisión sistemática de las revistas indexadas a junio 2020, disponibles en la base de datos de PubMed-Medline, ScienceDirect, con las palabras clave: anterior descending coronary superdominant e hyperdominant. Resultados: La presentación clínica de este caso hacía suponer obstrucciones en otros segmentos coronarios. La arteria descendente anterior superdominante justifica las alteraciones en cara inferior por las implicaciones anatómicas-funcionales. Al no encontrar lesiones obstructivas y no contar con estrategias específicas de tratamiento, el ajuste farmacológico parece ser una opción adecuada. Discusión: A pesar de ser una variante coronaria rara, las implicaciones clínicas se asocian no sólo al gran territorio que irriga y las consecuencias catastróficas en caso de obstrucción de la descendente anterior, sino también al compromiso anatómico y funcional por su longitud y disposición espacial que podría explicar una perfusión coronaria inferior anormal.

\subsection{Abuso de esteroides anabólicos: causa potencial de infarto agudo de miocardio en adulto joven}

Pacheco López Alejandro, * Pérez Rodríguez Eréndira Leticia, ${ }^{\ddagger}$ Garza Torres María del Pilar, ${ }^{\ddagger}$ García Rincón Andrés, ${ }^{\ddagger}$

Heredia Salazar Alberto Carlos ${ }^{\ddagger}$

* Hospital de Cardiología del Centro Médico Nacional

Siglo XXI. * Hospital San José Satélite.

Tipo de estudio: Reporte de casos clínicos con revisión de fuentes
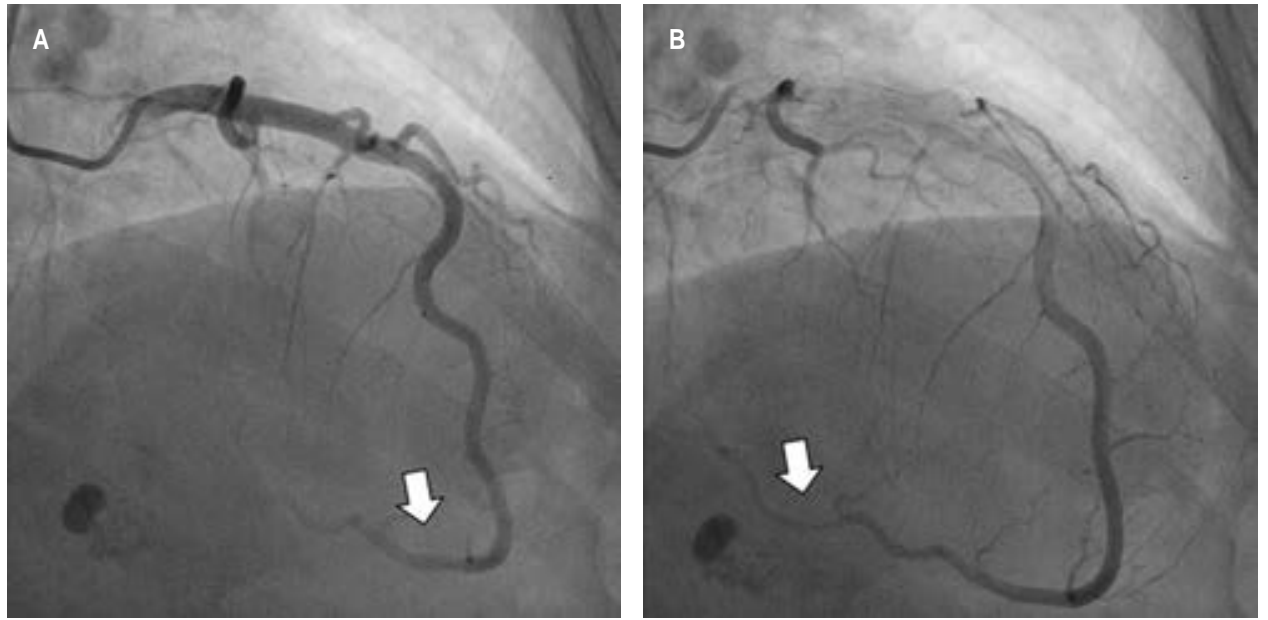

Figura 4.10.1:

A) Angiografía coronaria donde se observa la arteria descendente anterior superdominante que llega al ápex y continúa por el surco interventricular posterior hasta la cruz del corazón, originando desde el ápex la arteria descendente posterior. B) Se observa llenado tardío de la arteria descendente posterior que se origina de la descendente anterior (flecha). Arterias sin lesiones obstructivas. 
Figura 4.10.2:
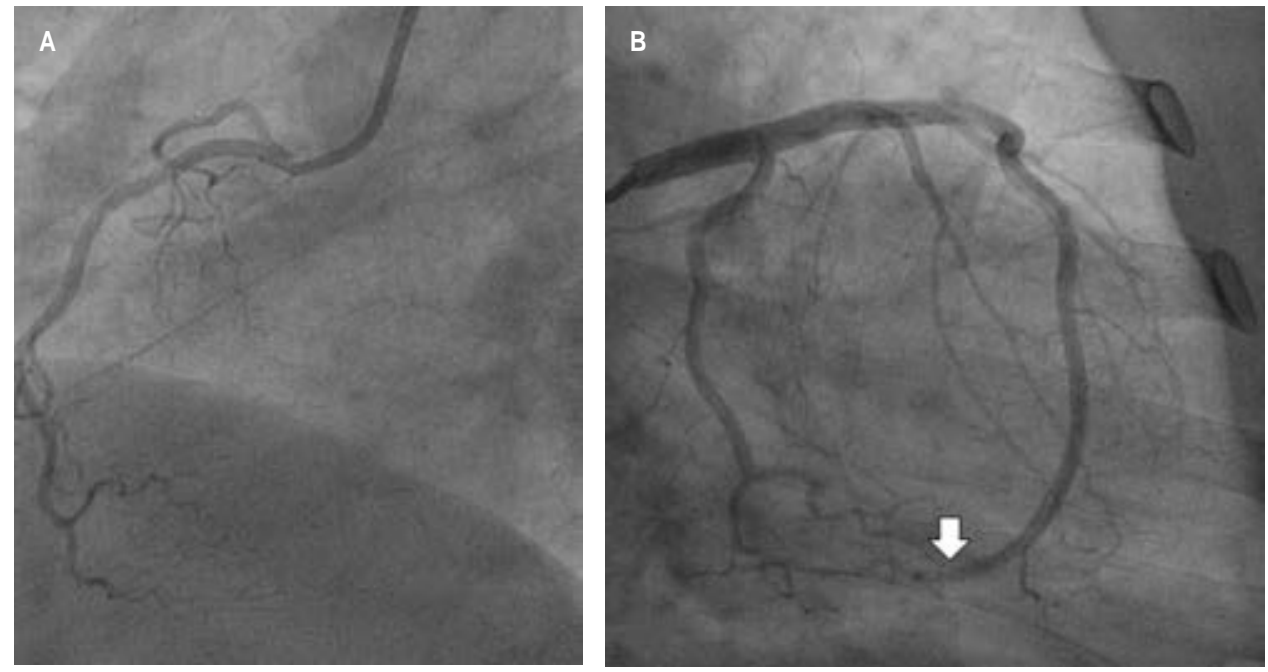

Introducción: En la mayoría de los casos de infarto del miocardio en jóvenes, factores de riesgo cardiovascular tradicionales siguen siendo importantes; en jóvenes se deben considerar otras causas, como el abuso de drogas; también los esteroides anabólicos derivados sintéticos de la testosterona, que los atletas usan ilegalmente para aumentar su rendimiento físico. Existe correlación entre el uso de estos fármacos con mayor riesgo cardiovascular. Descripción del caso: Hombre de 41 años, fisicoculturista con consumo crónico de esteroides anabólicos, 48 horas previas dolor torácico opresivo, arriba a urgencias con persistencia de sintomatología; electrocardiograma con bloqueo de rama derecha y cambios inespecíficos de repolarización; troponina I positiva; ecocardiograma: FEVI 59\% sin trastornos de movilidad; resonancia magnética infarto agudo de miocardio cara inferior transmural FEVI 49\%; se realiza intervencio-
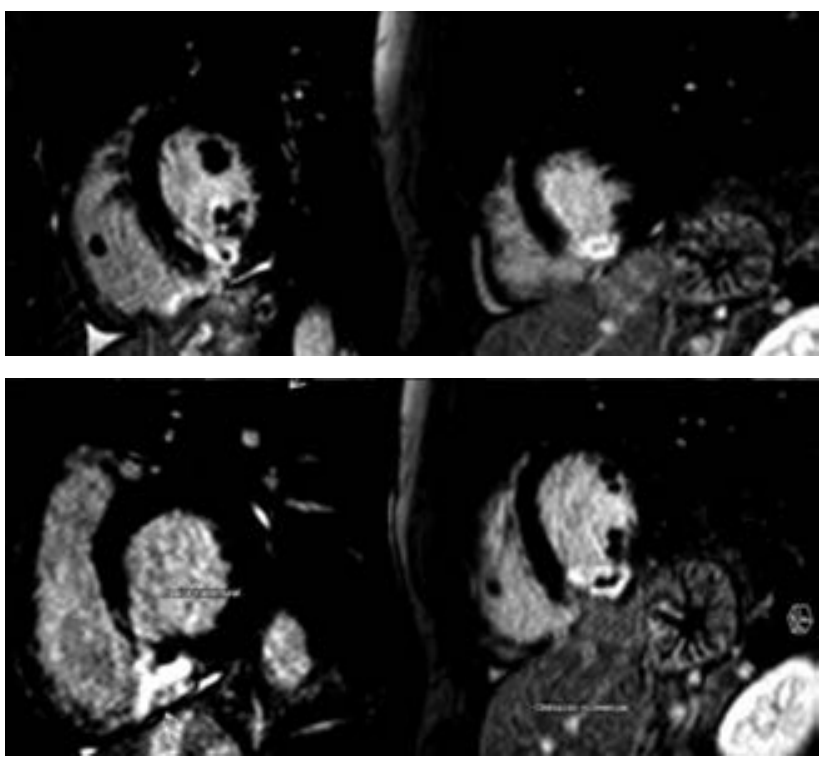

Figura 4.11.1. nismo coronario arteria coronaria derecha. Metodología: A través de método booleano se realizó búsqueda de términos MeSH myocardial infarction [and] anabolic steroid en las bases de datos con repositorios de evidencia científica arbitrada indexadas (PubMed, EBSCO, Ovid, ClinicalKey) y no indexadas (Google Scholar). Resultados: El abuso de esteroides anabólicos se ha asociado con disfunción ventricular tanto sistólica como diastólica. El abuso de éstos puede causar una lesión miocárdica directa que provoque un aumento de la deposición de colágeno, fibrosis y miocitólisis. Puede causar hipertrofia ventricular a través de la regulación positiva de los receptores de andrógenos y al final ateroesclerosis acelerada. Discusión: Es importante crear conciencia sobre los posibles efectos secundarios del uso de esteroides anabólicos de forma crónica, ya que pueden conducir al desarrollo de infarto agudo de miocardio. Una historia social detallada en la población de pacientes jóvenes puede cambiar las reglas del juego cuando se investigan las causas subyacentes del infarto del miocardio.

\subsection{Angiosarcoma de vena cava superior $y$ aurícula derecha. Reporte de un caso}

Romero Sánchez Javier Alberto, Ayón Ménez Vania Elizabeth, Becerril González Alejandro, Almeida Gutiérrez Eduardo, Lupercio Mora Karina

Hospital de Cardiología del Centro Médico Nacional Siglo XXI.

Tipo de estudio: Re irte de casos clínicos con revic" in de, ntes

Introducción: Los tumores cardiacos primarios generalmente son benignos. La etiología maligna es alrededor 25\%, localizado generalmente en aurícula derecha (AD) y de éstas 40\% son sarcomas, $75 \%$ en aurícula derecha. El angiosarcoma cardiaco es una neoplasia maligna rara, con metástasis temprana. La resonancia magnética es útil para el diagnóstico, para caracterización de infiltración de miocardio v pericardio. Descripción del caso: Hombre de 78 años. Tabac dismo, hipertensión arterial sistémica, síndrome de apnea-hipopnea obstructiva del sueño. Inicić $\mathrm{n}$ año previo con edema facial y miembros torácicos. Tomografía ce tórax: tumoración intracardiaca. Ecocardiograma transesofágico: masa intracardiaca, bordes irregulares, dependiente de vena cava superior (VCS) y cara anterosuperior de AD $5.9 \times 4.5 \mathrm{~cm}$. Tomografía abdominal: metástasis hepáticas. Resonancia magnética cardiaca: tumoración 
desde VCS hasta AD. Metodología: A través de método booleano se realizó búsqueda de términos MeSH tumoración auricular derecha y sarcoma auricular en las bases de datos con repositorios de evidencia científica arbitrada indizada (PubMed) y no indizadas (Google Scholar). Resultados: Las características por resonancia magnética cardiaca, hiperintensidad en secuencia T1 sin gadolinio y secuencia de reforzamiento tardío temprano con reforzamiento heterogéneo, así como por ecocardiografía son compatibles con angiosarcoma cardiaco. Cursa con lesiones hepáticas sugerentes de metástasis y alto riesgo quirúrgico. Discusión: El angiosarcoma cardiaco es una enfermedad rara, altamente invasiva, habitualmente con diagnóstico tardío, de difícil tratamiento quirúrgico, por lo cual los pacientes tienen supervivencia corta (media de 3.8 meses sin resección quirúrgica) y mal pronóstico.

\subsection{El papel de la cardiología nuclear en la prevención de la cardiotoxicidad}

Ávalos Ríos Javier Miguel,* Martínez Escobar María del Carmen,* Pérez Siller Grecia Rosangela, ${ }^{\ddagger}$ Escobedo Mercado Daniel,*

Gómez Leiva Verónica Vanesa, ${ }^{\S}$ Hernández Martínez Abraham A,* Mendoza Avellán Letty María*

* Centro Médico Nacional 20 de Noviembre, ISSSTE. * Hospital Regional «Dr. Valentín Gómez Farías». ${ }^{\S}$ Instituto Salvadoreño del Seguro Social. Tipo de estudio: Prueba diagnóstica

Introducción: Las enfermedades cardiovasculares y las neoplasias son las principales causas de mortalidad. Los pacientes con cáncer tienen hasta el doble de riesgo de padecer un síndrome coronario, la mayoría sin elevación del ST. Objetivo: Determinar

Figura 4.11.2.
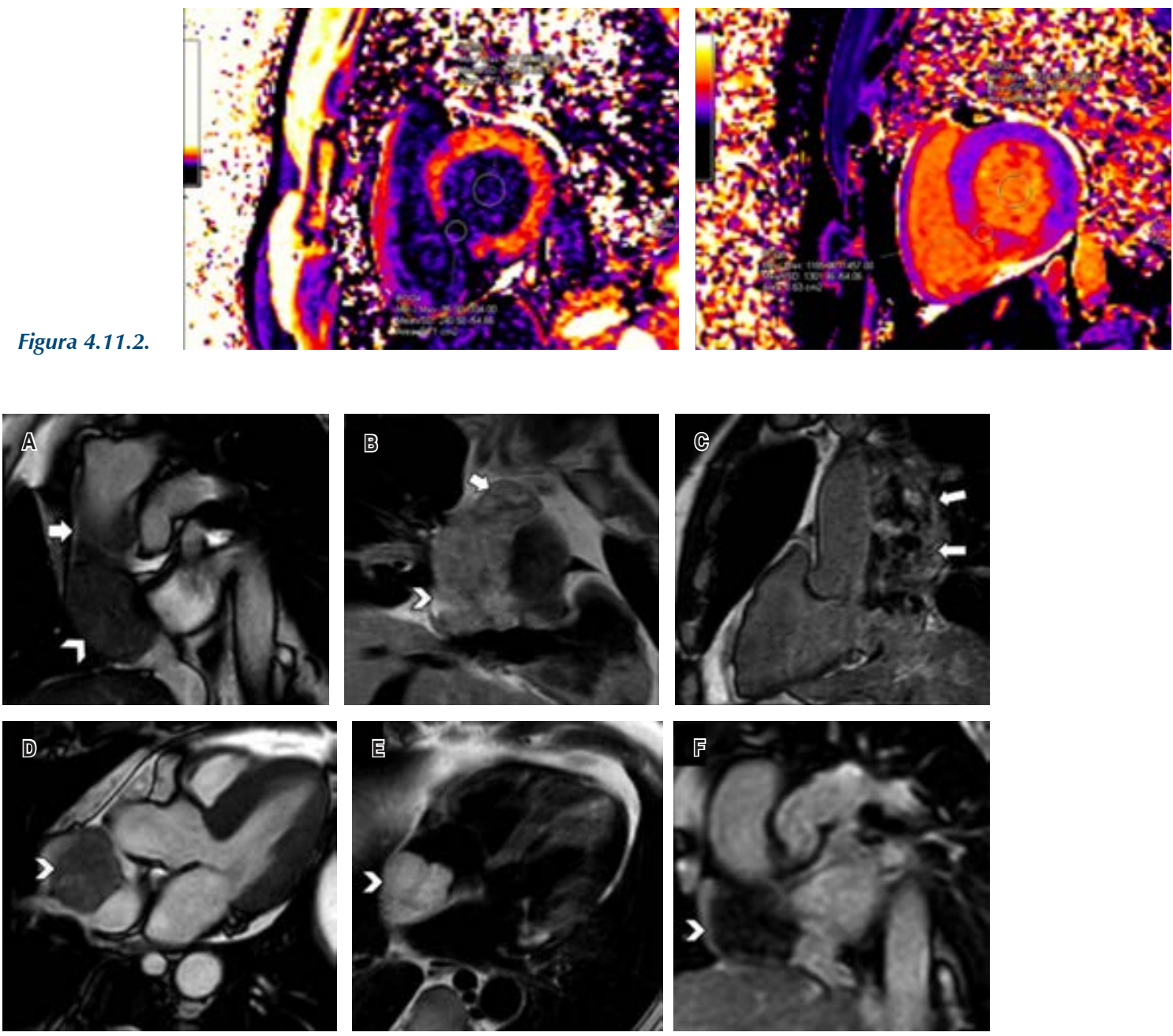

Figura 4.12.1: A) Imagen fija de una secuencia en cine en eje corto a nivel de la aurícula derecha. B) Imagen plano dos cámaras del ventrículo derecho ponderada en T1. C) Imagen de reforzamiento tardío de dos cámaras del ventrículo derecho. D) Imagen fija de secuencia de cine tracto de salida del ventrículo izquierdo. E) Imagen en cuatro cámaras ponderada en T2. F) Imagen fija de secuencia de primer paso eje corto de aurícula derecha. Las imágenes muestran una tumoración sólida (puntas de flecha) de márgenes lobulados que involucra el techo de la aurícula derecha con extensión a vena cava superior condicionando obstrucción total de la misma (flechas en $\boldsymbol{B}$ y $\boldsymbol{C}$ ). Presenta comportamiento hipointenso heterogéneo en T1 (B), heterogéneo hiperinteso en T2 (E), con reforzamiento heterogéneo en las secuencias de primer paso (F) y reforzamiento tardío (C). 

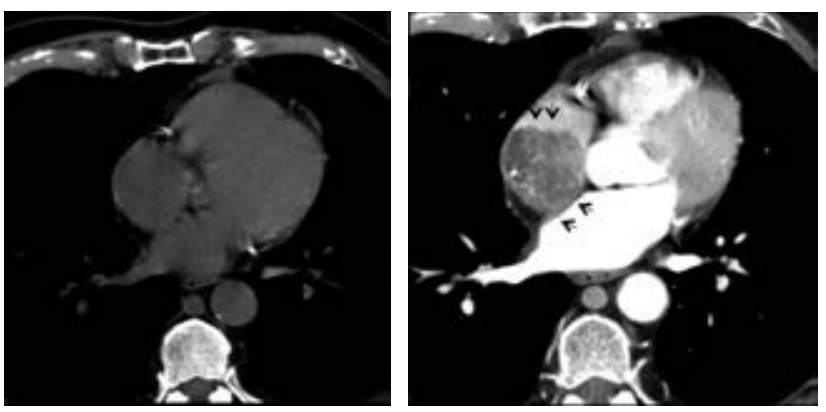

Figura 4.12.2: A) Cortes axiales de tomografía de tórax en fase simple y $\boldsymbol{B})$ con medio de contraste endovenoso, las cuales muestran un defecto de llenado sólido en la aurícula derecha (flechas en $\boldsymbol{B}$ ), de márgenes lobulados de comportamiento hipodenso homogéneo en fase simple (A) y con reforzamiento heterogéneo en fase contrastada (B) con imágenes hiperdensas lineales en su interior.

el rol de la cardiología nuclear en el diagnóstico de la cardiotoxicidad y su utilidad en pacientes con disnea o angina en pacientes con enfermedad oncológica. Metodología: Se ingresaron pacientes del Servicio de Oncología clínica o quirúrgica, con uso previo de quimioterapia, con síntomas tales como disnea o angina, a los cuales se les realizó estudio de perfusión miocár- dica, reposo-esfuerzo con TC-99. Resultados: Se enrolaron 14 pacientes. Neoplasia más frecuente fue cáncer de recto (37\%), $28.5 \%$ mujeres y $71.5 \%$ hombres. Edad promedio 60 años, $57 \%$ HAS, 29\% dislipidemia, 43\% DMT2, dos pacientes con infarto previo y tres con antecedente de angioplastia coronaria, 57\% presentaron defectos de perfusión ligeros y moderados; $50 \%$ riesgo ligero y $50 \%$ riesgo moderado, con FEVI mayor al 50\%. Un caso con cáncer de mama presentó FEVI menor al 30\%. Del grupo moderado se realizó cateterismo en dos pacientes, uno requirió angioplastia, el otro sin lesiones significativas. Discusión y conclusiones: Pacientes con enfermedad cardiovascular preexistente o factores de riesgo requieren de estratificación mediante gated-SPECT para establecer tratamiento durante antineoplásico, establecer pronóstico y monitoreo para prevenir eventos cardiovasculares mayores.

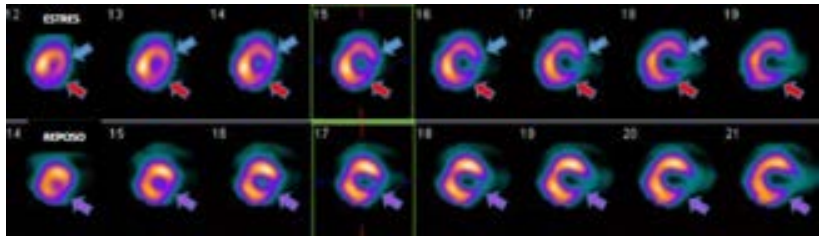

Figura 4.13.1: Imagen de perfusión miocárdica con infarto inferolateral e isquemia moderada e isquemia moderada anterolateral. 